Pacific Journal of Mathematics

THE SPECTRA OF ENDOMORPHISMS OF ALGEBRAS OF 


\title{
THE SPECTRA OF ENDOMORPHISMS OF ALGEBRAS OF ANALYTIC FUNCTIONS
}

\author{
Herbert Kamowitz
}

Suppose $0<R<1, G$ is the open annulus $\{z|R<| z \mid<1\}$ and $A(G)$ denotes the uniform algebra of functions analytic on $G$ and continuous on $\bar{G}$. Each nonzero endomorphism $T$ of $A(G)$ has the form $T f=f \circ \varphi$ for some $\varphi \in A(G)$ with $\varphi(G) \subset G$. In the main result of this note, the spectra of endomorphisms of $A(G)$ are determined for the case where the inducing maps $\varphi$ have a fixed point in $G$. In addition, further results are discussed for other algebras of analytic functions.

Introduction. In [2] we determined the spectra of a class of endomorphisms of the disc algebra $A(D)$, the uniform algebra of functions analytic on the open unit disc $D$ and continuous on $\bar{D}$. In this note, other algebras of analytic functions are considered and the techniques and results of [2] are used to prove a generalization of the following theorem.

THEOREM A. If $T$ is a nonzero endomorphism of $A(D)$, then Thas the form $T f=f \circ \varphi$, for some $\varphi \in A(D)$ with $\varphi: \bar{D} \rightarrow \bar{D}$. If, moreover, the inducing function $\varphi$ has a fixed point $z_{0}$ in $D$, then exactly one of the following three possibilities holds.

(1) $\varphi$ is a schlicht map of $D$ onto itself and $T$ is an automorphism of $A(D)$. In this case $\sigma(T)$, the spectrum of $T$, is either the entire unit circle or else $\sigma(T)$ is a finite union of finite subgroups of the circle, or

(2) $\sigma(T)=\{\lambda|| \lambda \mid \leqq 1\}$, or

(3) $T^{N}$ is a compact operator for some positive integer $N$ in which case $\sigma(T)=\left\{\left(\varphi^{\prime}\left(z_{0}\right)\right)^{n} \mid n\right.$ is a positive integer $\} \cup\{0,1\}$.

The plan is to first prove that an analytic function $\varphi$ which maps a bounded (open) region into itself has at most one fixed point unless $\varphi$ is schlicht and onto. Knowing this, we consider an annular region $G$ and the uniform algebra $A(G)$ of analytic functions on $G$ which are continuous on $\bar{G}$ and prove a theorem for $A(G)$ similar to Theorem A. Finally, we will indicate other regions for which similar results are valid and also state some later results concerning endomorphisms of the disc algebra.

1. Maps with two fixed points. In this section we prove 
that if $\varphi$ is an analytic function mapping a bounded region $\Omega$ into itself and if $\varphi$ has two fixed points in $\Omega$, then $\varphi$ is a schlicht map of $\Omega$ onto $\Omega$.

Throughout this section, $\Omega$ will denote a fixed bounded region (open connected subset) in the plane, and if $\varphi: \Omega \rightarrow \Omega$, then $\varphi_{n}$ will denote the $n$th iterate of $\varphi$, i.e. $\varphi_{1}=\varphi$ and $\varphi_{n}=\varphi \circ \varphi_{n-1}$.

Lemma 1.1. Suppose $\varphi$ is an analytic function, $\varphi: \Omega \rightarrow \Omega$ and $z_{0} \in \Omega$ is a fixed point of $\varphi$.

(i) If $\varphi^{\prime}\left(z_{0}\right)=1$, then $\varphi(z)=z$ for all $z \in \Omega$.

(ii) If $\varphi^{\prime}\left(z_{0}\right)^{N}=1$ for some positive integer $N$, then $\varphi_{N}(z)=z$ for all $z \in \Omega$.

Proof. (i) Suppose in a neighborhood of $z_{0}$ the function $\varphi$ has a Taylor series representation $\varphi(z)=z+c_{m}\left(z-z_{0}\right)^{m}+\cdots$. Since $\varphi(z)-z_{0}=z-z_{0}+c_{m}\left(z-z_{0}\right)^{m}+\cdots$, the Taylor series expansion of $\varphi_{2}$ at $z_{0}$ is $\varphi_{2}(z)=z+2 c_{m}\left(z-z_{0}\right)^{m}+\cdots$ and, in general, $\varphi_{n}(z)=$ $z+n c_{m}\left(z-z_{0}\right)^{m}+\cdots$. The $m$ th derivative of $\varphi_{n}$ at $z_{0}$ is $\varphi_{n}^{(m)}\left(z_{0}\right)=$ $n m ! c_{m}$.

On the other hand, since $\Omega$ is bounded, $\left\{\varphi_{n}\right\}$ is a normal family on $\Omega$ and so there exist an analytic function $\Phi$ on $\Omega$ and a subsequence $\left\{\varphi_{n_{k}}\right\}$ with $\varphi_{n_{k}} \rightarrow \Phi$ uniformly on compact subsets of $\Omega$. Since $\left\{\varphi_{n_{k}}^{(m)}\left(z_{0}\right)\right\}$ is unbounded unless $c_{m}=0$ and $\lim _{k \rightarrow x} \varphi_{n_{k}}^{(m)}\left(z_{0}\right)=\Phi^{(m)}\left(z_{0}\right)$, we conclude that $c_{m}=0$ and hence $\varphi(z)=z$ for all $z$ in a neighborhood of $z_{0}$. Since $\Omega$ is connected, $\varphi(z)=z$ for all $z \in \Omega$.

(ii) If $\varphi^{\prime}\left(z_{0}\right)^{N}=1$ for some positive integer $N$, then $\varphi_{N}^{\prime}\left(z_{0}\right)=\varphi^{\prime}\left(z_{0}\right)^{N}=$ 1 , so that $\varphi_{N}(z)=z$ for all $z \in \Omega$.

Lemma 1.2. Suppose $\varphi$ is an analytic function, $\varphi: \Omega \rightarrow \Omega$ and $z_{0} \in \Omega$ is a fixed point of $\varphi$. If $\left|\varphi^{\prime}\left(z_{0}\right)\right|=1$, then $\varphi$ is a schlicht map of $\Omega$ onto itself.

Proof. By Lemma 1.1 we need only prove this for the case where $\varphi^{\prime}\left(z_{0}\right)=e^{i \theta}$ and $e^{i \theta}$ is not a root of unity. For this case, let $\left\{n_{k}\right\}$ be an increasing sequence of positive integers with $e^{i n_{k} \theta} \rightarrow 1$. Again, $\left\{\varphi_{n_{k}}\right\}$ is a normal family on $\Omega$ and so there exist a subsequence $\left\{\varphi_{n_{k}}\right\}$ and an analytic function $\Phi$ on $\Omega$ with $\varphi_{n_{k j}} \rightarrow \Phi$ uniformly on compact subsets of $\Omega$.

Clearly $\Phi\left(z_{0}\right)=\lim _{j \rightarrow \infty} \varphi_{n_{k}}\left(z_{0}\right)=z_{0} \quad$ and $\quad \Phi^{\prime}\left(z_{0}\right)=\lim _{j \rightarrow \infty} \varphi_{n_{k}}^{\prime}\left(z_{0}\right)=$ $\lim _{j \rightarrow \infty} e^{i n_{k_{j}} \theta}=1$. Now consider the normal family $\left\{\varphi_{n_{k j}} \circ \varphi_{n_{k}}\right\}$. A routine calculation shows that $\left(\varphi_{n_{k j}} \circ \varphi_{n_{k j}}\right)^{(\nu)}\left(z_{0}\right) \rightarrow(\Phi \circ \Phi)^{(\nu)}\left(z_{0}\right)$ for each nonnegative integer $\nu$. On the other hand, there is a subsequence which we also call $\left\{\varphi_{n_{k j}} \circ \varphi_{n_{k}}\right\}$ and an analytic function $\psi: \Omega \rightarrow \Omega$ with $\varphi_{n_{k j}} \circ \varphi_{n_{k j}} \rightarrow \psi$ uniformly on compact sets. Also $\psi^{(\nu)}\left(z_{0}\right)=\lim _{j \rightarrow \infty}\left(\varphi_{n_{k j}}{ }^{\circ} \varphi_{n_{k j}}\right)^{(\nu)}\left(z_{0}\right)$ for nonnegative integers $\nu$. Thus $\Phi \circ \Phi=\psi$ near $z_{0}$ and so $\Phi \circ \Phi$ may be 
defined on all of $\Omega$ with $\Phi \circ \Phi: \Omega \rightarrow \bar{\Omega}$. In a similar way, for each positive integer $N, \Phi_{N}$ may be defined on $\Omega$ with range $\Phi_{N} \subset \bar{\Omega}$. Since $\Phi^{\prime}\left(z_{0}\right)=1$, by Lemma $1.1, \Phi(z)=z$ for all $z \in \Omega$. We have thus shown that $\lim _{ر \rightarrow \infty} \varphi_{n_{1}}(z)=z$ uniformly on compact subsets of $\Omega$.

The function $\varphi$ is schlicht since if $u, v \in \Omega$ and $\varphi(u)=\varphi(v)$, then $u=\Phi(u)=\lim _{ر \rightarrow \infty} \varphi_{n_{k j}}(u)=\lim _{\jmath \rightarrow \infty} \varphi_{n_{k}}(v)=\Phi(v)=v$.

To show that $\varphi$ is onto, suppose the contrary that for some $c \in \Omega$, $c \notin$ range $\varphi$. Suppose $r>0, C=\{z|| z-c \mid \leqq r\} \subset \Omega$ and $\gamma$ denotes the circle $\{z|| z-c \mid=r\}$. Since $c \notin$ range $\varphi$, for each positive integer $n$ and each $\quad z \in \Omega, \quad \varphi_{n}(z) \neq c$. Consider $\int_{\gamma}\left[\varphi_{n}^{\prime}(t) /\left(\varphi_{n}(t)-c\right)\right] d t$. Since $\varphi_{n}(t) \neq c$ for all $t \in C, \int_{\gamma}\left[\varphi_{n}^{\prime}(t) /\left(\varphi_{n}(t)-c\right)\right] d t=0$ for each $n$. On the other hand, we have shown that there is a subsequence $\left\{\varphi_{n_{k}}\right\}$ with $\varphi_{n_{k}} \rightarrow z$ uniformly on $C$. Hence, $0=\int_{\gamma}\left[\varphi_{n_{k}}^{\prime}(t) /\left(\varphi_{n_{k}}(t)-c\right)\right] d t \rightarrow \int_{\gamma}(t-c)^{-1} d t$ while $\int_{\gamma}(t-c)^{-1} d t=2 \pi i$, a contradiction. Thus, for some $t^{\prime} \in \Omega$ and some positive integer $n, \varphi_{n}\left(t^{\prime}\right)=c$ and so $c=\varphi\left(\varphi_{n-1}\left(t^{\prime}\right)\right)$. Thus $c \in$ range $\varphi$. Since $c$ is an arbitrary element in $\Omega$, we conclude that $\varphi$ is onto.

Combining with Lemma 1.1, we have shown that if $\varphi$ is an analytic function from $\Omega$ to $\Omega$ with a fixed point $z_{0}$ and if $\left|\varphi^{\prime}\left(z_{0}\right)\right|=1$, then $\varphi$ is a schlicht map of $\Omega$ onto itself.

LEMMA 1.3. Let $\varphi: \Omega \rightarrow \Omega$ be analytic with fixed point $z_{0}$ and suppose $\left|\varphi^{\prime}\left(z_{0}\right)\right|<1$. Then there exists a neighborhood $U$ of $z_{0}$ such that $\lim _{n \rightarrow \infty}\left|\varphi_{n}(z)-z_{0}\right|^{1 / n} \leqq\left|\varphi^{\prime}\left(z_{0}\right)\right|$ for $z \in U$ and consequently $\lim _{n \rightarrow \infty} \varphi(z)=$ $z_{0}$ for $z \in U$.

Proof. Choose $\epsilon>0$ satisfying $\left(\left|\varphi^{\prime}\left(z_{0}\right)\right|+\epsilon\right)<1$. Then there exists $\delta>0$ such that $0<\left|z-z_{0}\right|<\delta$ implies

$$
\left|\frac{\varphi(z)-z_{0}}{z-z_{0}}-\varphi^{\prime}\left(z_{0}\right)\right|<\epsilon .
$$

Hence, $\left|\varphi(z)-z_{0}\right|<\left(\left|\varphi^{\prime}\left(z_{0}\right)\right|+\epsilon\right)\left|z-z_{0}\right|$ for $\left|z-z_{0}\right|<\delta$. Then

$$
\left|\varphi(\varphi(z))-z_{0}\right|<\left(\left|\varphi^{\prime}\left(z_{0}\right)\right|+\epsilon\right)\left|\varphi(z)-z_{0}\right|<\left(\left|\varphi^{\prime}\left(z_{0}\right)\right|+\epsilon\right)^{2}\left|z-z_{0}\right|
$$

for $\left|z-z_{0}\right|<\delta$ and, by induction, $\left|\varphi_{n}(z)-z_{0}\right|<\left(\left|\varphi^{\prime}\left(z_{0}\right)\right|+\epsilon\right)^{n}\left|z-z_{0}\right|$ for all positive integers $n$ and $\left|z-z_{0}\right|<\delta$. Thus $\underline{\lim }_{n \rightarrow \infty}\left|\varphi_{n}(z)-z_{0}\right|^{1 / n} \leqq$ $\left|\varphi^{\prime}\left(z_{0}\right)\right|+\epsilon$ for all $\epsilon>0,\left|z-z_{0}\right|<\delta$ and so $\varlimsup_{\lim }\left|\varphi_{n}(z)-z_{0}\right|^{1 / n} \leqq$ $\left|\varphi^{\prime}\left(z_{0}\right)\right|$ for $\left|z-z_{0}\right|<\delta$. 
The statement concerning $\lim _{n \rightarrow x} \varphi_{n}(z)=z_{0}$ follows immediately.

THEOREM 1.4. Let $\Omega$ be a bounded region in the complex plane and $\varphi$ an analytic function from $\Omega$ into itself. If $\varphi$ has two fixed points in $\Omega$, then $\varphi$ is a schlicht map of $\Omega$ onto itself.

Proof. Suppose $z_{0}$ is a fixed point of $\varphi$. As we have seen, if $\left|\varphi^{\prime}\left(z_{0}\right)\right|=1$, then $\varphi$ is schlicht and onto.

The remainder of the proof is in two parts. We first show that $\left|\varphi^{\prime}\left(z_{0}\right)\right| \leqq 1$, and then show that if $\left|\varphi^{\prime}\left(z_{0}\right)\right|<1$, then $z_{0}$ is the only fixed point of $\varphi$.

Indeed, suppose $\left|\varphi^{\prime}\left(z_{0}\right)\right|>1$. If $\left\{\varphi_{n_{k}}\right\}$ is a subsequence converging uniformly on compact sets to the analytic function $\Phi$, then $\varphi_{n_{k}}^{\prime}\left(z_{0}\right) \rightarrow \Phi^{\prime}\left(z_{0}\right)$. But $\left\{\varphi_{n_{k}}^{\prime}\left(z_{0}\right)\right\}$ is unbounded since $\varphi_{n_{k}}^{\prime}\left(z_{0}\right)=\left(\varphi^{\prime}\left(z_{0}\right)\right)^{n_{k}}$.

For the other case, suppose $\left|\varphi^{\prime}\left(z_{0}\right)\right|<1$. If $\Phi$ is analytic on $\Omega$ and $\varphi_{n_{k}} \rightarrow \Phi$ uniformly on compact sets, then for each $z \in \Omega, \lim _{k \rightarrow \infty} \varphi_{n_{k}}(z)=$ $\Phi(z)$. But Lemma 1.3 implies that for $z$ near $z_{0}, \lim _{k \rightarrow x} \varphi_{n}(z)=$ $z_{0}$. Hence $\Phi(z)=z_{0}$ in a neighborhood of $z_{0}$ and so $\Phi(z)=z_{0}$ on $\Omega$. This implies that $z_{0}$ is the only fixed point of $\varphi$, since $\varphi(b)=b$ implies $b=\Phi(b)=z_{0}$.

REMARKs. (1) More is known if $\Omega$ is conformally equivalent to the disc. Then the only analytic map of $\Omega$ into itself with two fixed points is the identity. This can easily be proved by reducing to the case that $\Omega$ is the unit disc and using Schwarz Lemma.

(2) In the case of an annulus $G=\left\{z\left|r_{1}<\right| z \mid<r_{2}\right\}$, it can be shown using, for example, the Hadamard three circles theorem, that the only analytic maps of $G$ onto itself are linear fractional transformations which are either rotations about the origin (no fixed points unless the identity) or those of the form $\varphi(z)=e^{i \theta} r_{1} r_{2} / z$. In the latter case the fixed points are $\pm e^{\frac{1}{2} \theta \theta} \sqrt{r_{1} r_{2}}$ and $\varphi^{\prime}$ at each fixed point is -1 .

(3) The conclusion of Theorem 1.4 holds for any region which is conformally equivalent to a bounded region.

2. The annulus algebra. We now specialize and let $G$ be the open annulus $G=\{z|R<| z \mid<1\}$ where $0<R<1$, and let $A(G)$ denote the uniform algebra of functions analytic on $G$ and continuous on $\bar{G}$. For this algebra, too, every nonzero endomorphism $T$ is a composition operator of the form $T f=f \circ \varphi$ where $\varphi \in A(G)$ and $\varphi: \bar{G} \rightarrow \bar{G}$. In this section, the spectrum of $T$ is determined in the case $\varphi$ has a fixed point in the open set $G$.

LEMMA 2.1. If $T$ is a nonzero endomorphism of $A(G)$, then $T f=$ 
$f \circ \varphi$ where $\varphi=T z, z$ the identity function on $G$. The function $\varphi$ is in $A(G)$ and $\varphi: \bar{G} \rightarrow \bar{G}$.

Proof. Since $T$ is a nonzero endomorphism of a uniform algebra, it follows that $\|T\|=1$. Hence $\|T z\|_{\infty} \leqq\|T\|\|z\|_{\infty}=1$. Thus, if $\varphi=$ $T z \in A(G)$, then $\sup _{z \in G}|\varphi(z)|=\|\varphi\|_{\infty}=\|T z\|_{\infty} \leqq 1$. Also, $R / z \in A(G)$ and $\|R / z\|_{\infty}=1$. Therefore, $\|T(R / z)\|_{\infty} \leqq 1$. But $T(R / z)=R / \varphi(z)$. Thus, $\sup _{z \in G}|R / \varphi(z)| \leqq 1$ and hence $\inf _{z \in G}|\varphi(z)| \geqq R$, i.e. for $z \in G$, we have $R \leqq|\varphi(z)| \leqq 1$ and so $\varphi: \bar{G} \rightarrow \bar{G}$.

Further, each $f \in A(G)$ is a uniform limit of rational functions of the form $\sum_{k=-m}^{n} a_{k} z^{k}$. But for such a function $g: g(z)=\sum_{k=-m}^{n} a_{k} z^{k}$, we have

$$
(T g)(z)=T\left(\sum_{k=-m}^{n} a_{k} z^{k}\right)=\sum_{k=-m}^{n} a_{k}(T z)^{k}=\sum_{k=-m}^{n} a_{k}(\varphi(z))^{k}=g(\varphi(z)) .
$$

Hence $T f=f \circ \varphi$ for all $f \in A(G)$.

Conversely, for each $\varphi \in A(G)$ with $\varphi: \bar{G} \rightarrow \bar{G}$, the map $f \rightarrow f \circ \varphi$, for $f \in A(G)$, is a nonzero endomorphism of $A(G)$. Henceforth in this paper we will show the dependence of this endomorphism on the inducing function $\varphi$ by denoting the endomorphism by $C_{\varphi}$. Thus $\left(C_{\varphi} f\right)(z)=f(\varphi(z))$. It should be remarked that this is also standard notation for a composition operator.

[4]

Lemma 2.2. Let $\varphi \in A(G), \varphi: G \rightarrow G$ and let $z_{0} \in G$ be a fixed point of $\varphi$. Then the following hold.

(i) Suppose $\lambda \neq 0,1,\left(\varphi^{\prime}\left(z_{0}\right)\right)^{n}, n$ a positive integer and that $f, g \in$ $A(G)$ with $\lambda f-f \circ \varphi=g$. If $g$ has a zero of order $\nu$ at $z_{0}$, then $f$ has a zero of order at least $\nu$ at $z_{0}$.

(ii) If $\lambda \neq 0,1,\left(\varphi^{\prime}\left(z_{0}\right)\right)^{n}, n$ a positive integer, $f \in A(G)$ and $\lambda f(z)=$ $f(\varphi(z))$ in some neighborhood of $z_{0}$, then $f=0$.

(iii) The only possible eigenvalues of $C_{\varphi}$ are 0,1 or $\left(\varphi^{\prime}\left(z_{0}\right)\right)^{n}, n$ a positive integer.

(iv) $1 \in \sigma\left(C_{\varphi}\right)$, and for each positive integer $n,\left(\varphi^{\prime}\left(z_{0}\right)\right)^{n} \in$ $\sigma\left(C_{\varphi}\right)$. If $\varphi$ is not schlicht and onto, then $C_{\varphi}$ is not an automorphism and $0 \in \sigma\left(C_{\varphi}\right)$.

Proof. (i) This is essentially Lemma 3 in [2].

(ii) Evaluating the successive derivatives of $\lambda f(z)=f(\varphi(z))$ at $z_{0}$ gives that $f^{(\nu)}\left(z_{0}\right)=0$ for all nonnegative integers $\nu$ which implies that $f=0$.

(iii) This follows from (ii).

(iv) This is essentially Lemma 2 in [2]. The proof consists in 
showing that for each nonnegative integer $\nu$ there is no $f \in A(G)$ with $\left(\varphi^{\prime}\left(z_{0}\right)\right)^{\nu} f-f \circ \varphi=\left(z-z_{0}\right)^{\nu}$.

LemMA 2.3. Suppose $f$ and $g$ are defined on $G, \varphi: G \rightarrow G$ and $\lambda f-f \circ \varphi=g$. Then the following hold for every positive integer $n$.

(i) $f(z)=f\left(\varphi_{n}(z)\right) \lambda^{-n}+\sum_{k=0}^{n-1} g\left(\varphi_{k}(z)\right) \lambda^{-k-1}$ for all $z \in G$.

(ii) If $\left\{x_{k}\right\}_{k=-\infty}^{\infty}$ has the property that $\varphi\left(x_{p}\right)=x_{p+1}$ for all integers $p$, and $\varphi_{p}\left(x_{0}\right)=x_{p}, p>0$, then

$$
\lambda^{n} f\left(x_{-n}\right)=f\left(x_{n}\right) \lambda^{-n}+\sum_{k=-n}^{n-1} g\left(x_{k}\right) \lambda^{-k-1} .
$$

Proof. The proof by induction of (i) is in Lemma 5 in [2].

As for (ii), suppose $\left\{x_{n}\right\}_{n=-\infty}^{\infty}$ has the property that $\varphi\left(x_{p}\right)=x_{p+1}$ for all integers $p$ and $\varphi_{p}\left(x_{0}\right)=x_{p}, p>0$. Then, from (i),

$$
\lambda^{2 n} f(z)=f\left(\varphi_{2 n}(z)\right)+\sum_{k=0}^{2 n-1} g\left(\varphi_{k}(z)\right) \lambda^{2 n-k-1} .
$$

Thus if we let $z=x_{-n}$, we obtain

$$
\lambda^{2 n} f\left(x_{-n}\right)=f\left(x_{n}\right)+\sum_{k=0}^{2 n-1} g\left(x_{k-n}\right) \lambda^{2 n-k-1}
$$

Hence

$$
\lambda^{n} f\left(x_{-n}\right)=f\left(x_{n}\right) \lambda^{-n}+\sum_{k=0}^{2 n-1} g\left(x_{k-n}\right) \lambda^{n-k-1}
$$

which is equivalent to $(*)$.

LemMA 2.4. Suppose $T$ is a bounded linear operator on a Banach space with the property that for some number $a,|a| \leqq 1$, we have

(i) $\sigma(T) \supset\left\{a^{n} \mid n\right.$ is a positive integer $\} \cup\{0,1\}$, and

(ii) for some positive integer $N, \sigma\left(T^{N}\right)=\left\{a^{N k} \mid k\right.$ is a positive integer $\} \cup\{0,1\}$ and $\sigma\left(T^{N+1}\right)=\left\{a^{(N+1) k} \mid k\right.$ is a positive integer $\} \cup\{0,1\}$.

Then $\sigma(T)=\left\{a^{n} \mid n\right.$ is a positive integer $\} \cup\{0,1\}$.

Proof. Suppose $0 \neq \lambda \in \sigma(T)$. Then $\lambda^{N} \in \sigma\left(T^{N}\right)$ and $\lambda^{N+1} \in$ $\sigma\left(T^{N+1}\right)$, and so (ii) implies there exist positive integers $j$ and $k$ for which $\lambda^{N}=a^{N_{j}}$ and $\lambda^{N+1}=a^{(N+1) k}$. Therefore, $|\lambda|=|a|^{j}$ and $|\lambda|=|a|^{k}$ showing that $j=k$. Hence $\lambda=a^{k}$ for some positive integer $k$. 
Thus $\sigma(T) \subset\left\{a^{k} \mid k\right.$ is a positive integer $\} \cup\{0,1\}$ which, together with (i), implies that $\sigma(T)=\left\{a^{k} \mid k\right.$ is a positive integer $\} \cup\{0,1\}$.

The main result will be proved in three parts, Theorems 2.5, 2.6 and 2.7 .

It was observed at the end of $\S 1$ that the only schlicht analytic maps of the open annulus $G$ onto itself have the forms $\varphi(z)=e^{1 \alpha} z, \alpha$ real, or $\varphi(z)=e^{i \alpha} R / z, \alpha$ real. Further, if $T$ is any automorphism of a commutative semi-simple Banach algebra, then it has been shown ([1], [3]) that either $T^{N}=I$ for some positive integer $N$ in which case $\sigma(T)$ is a finite union of finite subgroups of the circle, or else $\sigma(T)=\{\lambda|| \lambda \mid=1\}$. Thus we have the following.

THEOREM 2.5. If $0<R<1, G=\{z|R<| z \mid<1\}$ and $T$ is an automorphism of $A(G)$, then $T=C_{\varphi}$ where $\varphi \in A(G)$ is a schlicht map of $\bar{G}$ onto $\bar{G}$. Such $\varphi$ take the form $\varphi(z)=e^{i \alpha} z, \alpha$ real, or $\varphi(z)=e^{i \alpha} R / z, \alpha$ real. In the first case $\sigma\left(C_{\varphi}\right)=$ closure $\left\{e^{i n \alpha} \mid n\right.$ is a nonnegative integer $\}$, while if $\varphi(z)=e^{1 \alpha} R / z$, then $\sigma\left(C_{\varphi}\right)=\{-1,+1\}$.

For more general theorems concerning spectra of automorphisms of Banach algebras see [1] or [3].

Now suppose $\varphi \in A(G), \varphi: \bar{G} \rightarrow \bar{G}$ and $\varphi$ is not schlicht and onto. Let $S=\bigcap_{n=1}^{\infty} \varphi_{n}(\bar{G})$. A straightforward topological argument shows that $S$ is a nonempty compact connected subset of $\bar{G}$ and that $\varphi$ maps $S$ onto itself. Suppose $z_{0} \in G$ is a fixed point of $\varphi$. The results of $\$ 1$ show that this point is unique. Clearly $z_{0} \in S$ and if $S \neq\left\{z_{0}\right\}$, then the connected set $S$ is infinite. In this case there is a sequence $\left\{x_{n}\right\}_{n=-\infty}^{\infty} \subset S$ with $\varphi\left(x_{p}\right)=x_{p+1}$ for all integers $p$. To construct such a sequence, let $z_{0} \neq x_{0} \in S \cap G$ and define $x_{n}=\varphi_{n}\left(x_{0}\right) \in S \cap G$ for $n \geqq 0$ and for $n>0$ let $x_{-n} \in S$ satisfy $\varphi\left(x_{-n}\right)=x_{r_{n+1}}$. Although $x_{-n}$ need not be unique, we can find such $x_{-n}$ since $\varphi$ maps $S$ onto itself.

THEOREM 2.6. Let $0<R<1$ and $G=\{z|R<| z \mid<1\}$. Suppose $\varphi \in A(G), \varphi: G \rightarrow G, \varphi\left(z_{0}\right)=z_{0} \in G$ and $S=\bigcap_{n=1}^{\infty} \varphi_{n}(\bar{G})$ is infinite. If $C_{\varphi}$ is not an automorphism of $A(G)$, then $\sigma\left(C_{\varphi}\right)=\{\lambda|| \lambda \mid \leqq 1\}$.

Proof. Since $C_{\varphi}$ is not an automorphism, the inducing function $\varphi$ is not schlicht and onto and so it follows from Lemma 1.2 that $\left|\varphi^{\prime}\left(z_{0}\right)\right|<1$.

We will show that if $\nu$ is a positive integer, then $\sigma\left(C_{\varphi}\right) \supset$ $\left\{\left.\lambda|| \varphi^{\prime}\left(z_{0}\right)\right|^{\nu}<|\lambda|<1\right\}$. To this end fix $\nu$ and let $g: g(z)=$ $\left(z-z_{0}\right)^{\nu}$. Further, suppose $x_{0} \neq z_{0}, x_{0} \in S \cap G$ and that $\left\{x_{n}\right\}_{n=-\infty}^{\infty}$ is a sequence in $S$ satisfying $\varphi\left(x_{n}\right)=x_{n+1}$ for all integers $n$, and $x_{n}=\varphi_{n}\left(x_{0}\right)$ for 
$n \geqq 0$. Assume, too, that $x_{0}$ has been chosen close enough to $z_{0}$ that $\lim _{n \rightarrow \infty} \varphi_{n}\left(x_{0}\right)=z_{0}$.

Since $g$ has a zero of order $\nu$ at $z_{0}$, if $f \in A(G)$ and $\lambda f-f \circ \varphi=g$, then by Lemma 2.2(i), $f(z)=c_{\nu+p}\left(z-z_{0}\right)^{\nu+p}+\mathcal{O}\left(\left|z-z_{0}\right|^{\nu+p+1}\right)$ near $z_{0}$ for some integer $p \geqq 0$ and complex number $c_{\nu+p}$. Hence $f\left(\varphi_{n}(z)\right)=$ $c_{\nu+p}\left(\varphi_{n}(z)-z_{0}\right)^{\nu+p}+\mathscr{O}\left(\left|\varphi_{n}(z)-z_{0}\right|^{\nu+p+1}\right)$ for $z$ near $z_{0}$. Moreover, from Lemma 1.3 we have $\varlimsup_{\lim _{n \rightarrow \infty}}\left|\varphi_{n}(z)-z_{0}\right|^{1 / n} \leqq\left|\varphi^{\prime}\left(z_{0}\right)\right|$ for $z$ near $z_{0}$. Thus, for such $z$, if $\left|\varphi^{\prime}\left(z_{0}\right)\right|^{\nu}<|\lambda|$, then

$$
\varlimsup_{\lim }\left|f\left(\varphi_{n}(z)\right)\right||\lambda|^{-n} \leqq \varlimsup_{n \rightarrow \infty}\left|c_{\nu+p}\right|\left|\varphi_{n}(z)-z_{0}\right|^{\nu+p}|\lambda|^{-n}=0 .
$$

Now assume that $\sigma\left(C_{\varphi}\right)$ does not contain $\left\{\left.\lambda|| \varphi^{\prime}\left(z_{0}\right)\right|^{\nu}<|\lambda|<1\right\}$ and that $\lambda_{0} \notin \sigma\left(C_{\varphi}\right)$ with $\left|\varphi^{\prime}\left(z_{0}\right)\right|^{\nu}<\left|\lambda_{0}\right|<1$. Let $U$ be a neighborhood of $\lambda_{0}$ with $U \subset\left\{\left.\lambda|| \varphi^{\prime}\left(z_{0}\right)\right|^{\nu}<|\lambda|<1\right\}$, and $U \cap\left\{\left(\varphi^{\prime}\left(z_{0}\right)\right)^{n} \mid n\right.$ is a positive integer $\}=\varnothing$ and such that for each $\lambda \in U,\left(\lambda-C_{\varphi}\right)^{-1}$ exists. Then for each $\lambda \in U$ there exists a function $f_{\lambda} \in A(G)$ with $\lambda f_{\lambda}-f_{\lambda} \circ \varphi=g$.

By Lemma 2.3(ii), for each positive integer $n$, we have

$$
\lambda^{n} f_{\lambda}\left(x_{-n}\right)=f_{\lambda}\left(x_{n}\right) \lambda^{-n}+\sum_{k=-n}^{n-1} g\left(x_{k}\right) \lambda^{-k-1} \text {. }
$$

Also, since the map $\lambda \rightarrow\left(\lambda-C_{\varphi}\right)^{-1}$ is analytic on $U$, there exists a positive number $M$ such that $\left\|\left(\lambda-C_{\varphi}\right)^{-1}\right\| \leqq M$ for all $\lambda \in U$. Hence $\left\|f_{\lambda}\right\|_{\infty}=\left\|\left(\lambda-C_{\varphi}\right)^{-1} g\right\|_{\infty} \leqq M\|g\|_{\infty}$ for all $\lambda \in U$. Therefore, for $\lambda \in U$, $\lim _{n \rightarrow \infty} \lambda^{n} f_{\lambda}\left(x_{-n}\right)=0$. Since we have already shown that $\lim _{n \rightarrow \infty} f_{\lambda}\left(x_{n}\right) \lambda^{-n}=\lim _{n \rightarrow \infty} f_{\lambda}\left(\varphi_{n}\left(x_{0}\right)\right) \lambda^{-n}=0$, we have from $(*)$ that $\sum_{k=-\infty}^{\infty} g\left(x_{k}\right) \lambda^{-k}=0$ for all $\lambda$ in the open set $U \subset\left\{\left.\lambda|| \varphi^{\prime}\left(z_{0}\right)\right|^{\nu}<|\lambda|<1\right\}$.

However, the function $w \rightarrow \sum_{k=-\infty}^{\infty} g\left(x_{k}\right) w^{-k}$ is analytic in the annulus $\left\{\left.w|| \varphi^{\prime}\left(z_{0}\right)\right|^{\nu}<|w|<1\right\}$ since $\lim _{n \rightarrow \infty}\left|g\left(x_{-n}\right)\right|^{1 / n}=1$ and

$$
\varlimsup_{\lim _{k \rightarrow \infty}}\left|g\left(\varphi_{k}\left(x_{0}\right)\right)\right|^{1 / k}=\varlimsup_{\lim _{k \rightarrow \infty}}\left|\left(\varphi_{k}\left(x_{0}\right)-z_{0}\right)^{\nu}\right|^{1 / k} \leqq\left|\varphi^{\prime}\left(z_{0}\right)\right|^{\nu} .
$$

But, $\sum_{k=-\infty}^{\infty} g\left(x_{k}\right) \lambda^{-k}=0$ for $\lambda \in U$. Hence $\sum_{k=-\infty}^{\infty} g\left(x_{k}\right) w^{-k}=0$ for all $w$ with $\left|\varphi^{\prime}\left(z_{0}\right)\right|^{\nu}<|w|<1$. This implies that $g\left(x_{k}\right)=0$ for all integers $k$, which is a contradiction to the assumption that $g\left(x_{0}\right)=\left(x_{0}-z_{0}\right)^{\nu}$ and $x_{0} \neq z_{0}$. Hence $\sigma\left(C_{\varphi}\right) \supset\left\{\left.\lambda|| \varphi^{\prime}\left(z_{0}\right)\right|^{\nu}<|\lambda|<1\right\}$. Since this holds for all positive integers $\nu$, we conclude that $\sigma\left(C_{\varphi}\right)=\{\lambda|| \lambda \mid \leqq 1\}$, as desired.

For an arbitrary region $\Omega$ we let $A(\Omega)=\{f \mid f$ is analytic on $\Omega$ and continuous on $\bar{\Omega}\}$. If $\Omega$ is a simply connected Jordan region (a region whose boundary is a Jordan curve), then $A(\Omega)$ is isomorphic to the disc algebra $A(D)$. This follows from the fact that if $\beta$ is a conformal map of $\Omega$ onto $D$, then $\beta$ extends to a homeomorphism of $\bar{\Omega}$ onto $\bar{D}$. Further, if $T$ is a nonzero endomorphism of $A(\Omega)$, then $T f=f \circ \varphi$ for some $\varphi \in A(\Omega), \varphi: \bar{\Omega} \rightarrow \bar{\Omega}$. Moreover, if $\psi=\beta \circ \varphi \circ \beta^{-1}$, then 
$\psi \in A(D)$ with $\psi(\bar{D}) \subset \bar{D}$ and it is easy to verify that if $C_{\varphi}$ and $C_{\psi}$ are the linear operators induced by $\varphi$ and $\psi$ on $A(\Omega)$ and $A(D)$ respectively, then $\sigma\left(C_{\varphi}\right)=\sigma\left(C_{\psi}\right)$.

THEOrem 2.7. Let $0<R<1$ and $G=\{z|R<| z \mid<1\}$. If $\varphi: G \rightarrow G, \varphi \in A(G)$ and $S=\bigcap_{n=1}^{\infty} \varphi_{n}(\bar{G})=\left\{z_{0}\right\} \subset G$, then $\sigma\left(C_{\varphi}\right)=$ $\left\{\left(\varphi^{\prime}\left(z_{0}\right)\right)^{n} \mid n\right.$ is a positive integer $\} \cup\{0,1\}$.

Proof. Let $G_{1}$ and $G_{2}$ be two overlapping simply connected Jordan regions with $\bar{G}_{1} \cup \bar{G}_{2}=\bar{G}$. Specifically, let $\alpha_{1}=\frac{1}{2} \pi+\arg z_{0}$ and $\alpha_{2}=$ $-\frac{1}{2} \pi+\arg z_{0}$ and let $\delta=\pi / 100$. For $k=1,2$, let $G_{k}$ be the region bounded by Jordan curves each consisting of the following four arcs.

$\gamma_{k, 1}=$ radial segment from $\operatorname{Re}^{i \alpha_{k}}$ to $e^{i \alpha_{k}}$,

$\gamma_{k, 2}=$ clockwise circular arc from $e^{i \alpha_{k}}$ to $e^{-t\left(2 \pi-\alpha_{k}-\delta\right)}$,

$\gamma_{k, 3}=$ radial segment from $e^{-\left(2 \pi-\alpha_{k}-\delta\right) t}$ to $\mathrm{Re}^{-\left(2 \pi-\alpha_{k}-\delta\right) t}$,

$\gamma_{k, 4}=$ counterclockwise circular arc from $\mathrm{Re}^{-1\left(2 \pi-\alpha_{k}-\delta\right)}$ to $\mathrm{Re}^{i \alpha_{k}}$.

Choose $\epsilon>0$ so that $U=\left\{z|| z-z_{0} \mid<\epsilon\right\} \subset G_{1} \cap G_{2}$. Since $S=$ $\bigcap_{n=1}^{\infty} \varphi_{n}(\bar{G})=\left\{z_{0}\right\}$, there exists a positive integer $N$ for which $\varphi_{N+1}(\bar{G}) \subset$ $\varphi_{N}(\bar{G}) \subset U$. For this $N, \varphi_{N}\left(\bar{G}_{1}\right) \subset U \subset G_{1}$ and $\varphi_{N}\left(\bar{G}_{2}\right) \subset U \subset G_{2}$. The operator $C_{\varphi_{N}}=C_{\varphi}^{N}$ is then an endomorphism of both $A\left(G_{1}\right)$ and $A\left(G_{2}\right)$ and, further, since $\bigcap_{j=1}^{\infty} \varphi_{N_{j}}\left(\bar{G}_{k}\right)=\left\{z_{0}\right\}, k=1,2$, it follows that $\sigma\left(C_{\varphi_{N}}\right)=$ $\left\{\left(\varphi_{N}^{\prime}\left(z_{0}\right)\right)^{k} \mid k\right.$ is a positive integer $\} \cup\{0,1\}$ as an operator both on $A\left(G_{1}\right)$ and $A\left(G_{2}\right)$.

Thus, given $g \in A(G)$ and $\lambda \notin\left\{\left(\varphi^{\prime}\left(z_{0}\right)\right)^{N k} \mid k\right.$ is a positive integer $\} \cup$ $\{0,1\}$ there exist $f_{1} \in A\left(G_{1}\right)$ and $f_{2} \in A\left(G_{2}\right)$ with $\lambda f_{1}(z)-f_{1}\left(\varphi_{N}(z)\right)=g(z)$ for $z \in \bar{G}_{1}$ and $\lambda f_{2}(z)-f_{2}\left(\varphi_{N}(z)\right)=g(z)$ for $z \in \bar{G}_{2}$.

Now, on $U \subset G_{1} \cap G_{2}$,

$$
\lambda f_{1}(z)-f_{1}\left(\varphi_{N}(z)\right)=\lambda f_{2}(z)-f_{2}\left(\varphi_{N}(z)\right) .
$$

Then Lemma 2.2(ii) implies that $f_{1}(z)=f_{2}(z)$ on the component of $G_{1} \cap G_{2}$ which contains $z_{0}$.

On the other hand, for all $z \in G_{1} \cap G_{2}$ we have by Lemma 2.3(i) that

$$
f_{1}(z)=f_{1}\left(\varphi_{N}(z)\right) \lambda^{-N}+\sum_{k=0}^{N-1} g\left(\varphi_{k}(z)\right) \lambda^{-k-1}
$$

and

$$
f_{2}(z)=f_{2}\left(\varphi_{N}(z)\right) \lambda^{-N}+\sum_{k=0}^{N-1} g\left(\varphi_{k}(z)\right) \lambda^{-k-1}
$$

Since $\varphi_{N}(z) \in U$, it follows that $f_{1}\left(\varphi_{N}(z)\right)=f_{2}\left(\varphi_{N}(z)\right)$ and so $f_{1}(z)=f_{2}(z)$ on all of $G_{1} \cap G_{2}$. Since $f_{1}$ is continuous on $G_{1}, f_{2}$ is continuous on $G_{2}$ 
and $f_{1}=f_{2}$ on $\bar{G}_{1} \cap \bar{G}_{2}$, we have that $f=f_{1} \cup f_{2} \in A(G)$ and $\lambda f-f \circ \varphi=$ g. Therefore, if $\lambda \notin\left\{\left(\varphi_{N}^{\prime}\left(z_{0}\right)\right)^{k} \mid k\right.$ is a positive integer $\} \cup\{0,1\}$, then $\left(\lambda-C_{\varphi}\right)^{-1}$ exists on $A(G)$, i.e. $\sigma\left(C_{\varphi_{N}}\right) \subset\left\{\left(\varphi^{\prime}\left(z_{0}\right)\right)^{N k} \mid k\right.$ is a positive integer $\} \cup\{0,1\}$ as an operator on $A(G)$. However, Lemma 2.2(iv) implies that the opposite inclusion holds, and so $\sigma\left(C_{\varphi_{N}}\right)=\left\{\left(\varphi^{\prime}\left(z_{0}\right)\right)^{N k} \mid k\right.$ is a positive integer $\} \cup\{0,1\}$.

On the other hand, a similar result holds for $\sigma\left(C_{\varphi_{N+1}}\right)$ since $\varphi_{N+1}(\bar{G})$ is also a subset of $U$. That is, $\sigma\left(C_{\varphi_{N+1}}\right)=\left\{\left(\varphi^{\prime}\left(z_{0}\right)\right)^{(N+1) k} \mid k\right.$ is a positive integer $\} \cup\{0,1\}$. Therefore, by Lemma 2.4, we conclude that $\sigma\left(C_{\varphi}\right)=$ $\left\{\left(\varphi^{\prime}\left(z_{0}\right)\right)^{n} \mid n\right.$ is a positive integer $\} \cup\{0,1\}$ as required.

To summarize, Theorems 2.5, 2.6 and 2.7, combined, show that Theorem $\mathrm{A}$ is valid for the annulus algebra.

3. Final remarks. The theorems in $\$ 2$ can be extended further to composition operators on uniform algebras of analytic functions on regions other than the disc or the annulus. The analogues of Theorems 2.5 and 2.6 can be proved in exactly the same manner as the originals. In Theorem 2.7, a crucial part of the proof was to express $G$ as a union of two overlapping simply connected Jordan regions. Thus if $\Omega$ is conformally equivalent to any bounded region which can be written as a union of two overlapping simply connected Jordan regions and if $\varphi \in A(\Omega), \varphi: \Omega \rightarrow \Omega$ and if $\varphi\left(z_{0}\right)=z_{0} \in \Omega$, then Theorems 2.5, 2.6 and 2.7 hold with $G$ replaced by $\Omega$.

A final remark relates to the disc algebra and the case where $\varphi$ has all its fixed points on the circle. In this case the spectrum of $C_{\varphi}$ need not have the same form as the operators in Theorem A. Indeed, if $\varphi$ is the linear fractional transformation $\varphi(z)=(z+1) /(3-z)$, then D. J. Newman (personal communication) has shown that $\sigma\left(C_{\varphi}\right)=[0,1]$, the unit interval. A slight modification of this example can be constructed for which $\sigma\left(C_{\varphi}\right)$ is the spiral $\{0\} \cup\left\{e^{-a t} \mid 0 \leqq t\right\}, a$ fixed with $\operatorname{Re} a>0$. This arises from the linear fractional transformation $\varphi(z)=$ $[(2-a) z+a] /[-a z+(a+2)]$.

\section{REFERENCES}

1. B. E. Johnson, Automorphisms of commutative Banach algebras, Proc. Amer. Math. Soc., 40 (1973), 497-499.

2. H. Kamowitz, The spectra of endomorphisms of the disc algebra, Pacific J. Math., 46 (1973), 433-440.

3. H. Kamowitz and S. Scheinberg, The spectrum of automorphisms of Banach algebras, J. Functional Analysis, 4 (1969), 268-276.

4. E. A. Nordgren, Composition operators, Canad. J. Math., 20 (1968), 442-449.

Received May 10, 1976.

UNIVERSITY OF MASSACHUSETTS AT Boston 


\section{PACIFIC JOURNAL OF MATHEMATICS}

\section{EDITORS}

RICHARI) ARENS (Managing Editor)

University of California

Los Angeles, CA 90024

R. A. Beaumiont

University of Washington

Seattle, WA 98105

\section{J. DugunduI}

Department of Mathematics University of Southern California Los Angeles, CA 90007

D. Gilbarg and J. Milgram Stanford University

Stanford, CA 94305

\section{ASSOCIATE EDITORS}

E. F. BECKENBACH
B. H. NEUMANN

F. Wolf

K. YoshidA

\section{SUPPORTING INSTITUTIONS}

UNIVERSITY OF BRITISH COLUMBIA CALIFORNIA INSTITUTE OF TECHNOLOGY

UNIVERSITY OF CALIFORNIA

MONTANA STATE UNIVERSITY

UNIVERSITY OF NEVADA

NEW MEXICO STATE UNIVERSITY

OREGON STATE UNIVERSITY

UNIVERSITY OF OREGON

OSAKA UNIVERSITY

\author{
UNIVERSITY OF SOUTHERN CALIFORNIA \\ STANFORD UNIVERSITY \\ UNIVERSITY OF HAWAII \\ UNIVERSITY OF TOKYO \\ UNIVERSITY OF UTAH \\ WASHINGTON STATE UNIVERSITY \\ UNIVERSITY OF WASHINGTON \\ AMERICAN MATHEMATICAL SOCIETY
}

The Supporting Institutions listed above contribute to the cost of publication of this Journal, but they are not owners or publishers and have no responsibility for its contents or policies.

Mathematical papers intended for publication in the Pacific Journal of Mathematics should be in typed form or offset-reproduced (not dittoed), double spaced with large margins. Underline Greek letters in red, German in green, and script in blue. The first $p: 1<$ graph or two must be capable of being used separately as a synopsis of the entire paper. Items of the biblography should not be cited there unless absolutely necessary, in which case they must he identified by author and Journal, rather than by item number. Manuscripts, in duplicate, may be sent to any one of the four editors. Please classify according to the scheme of Math. Reviews, Index to Vol. 39. All other communications should be addressed to the managing editor, or Elaine Barth, University of California, Los Angeles, California, 90024.

100 reprints are provided free for each article, only if page charges have been substantially paid. Additional copies may be obtained at cost in multiples of 50 .

The Pacific Journal of Mathematics is issued monthly as of January 1966. Regular subscription rate: $\$ 72.00$ a year (6 Vols., 12 issues). Special rate: $\$ 36.00$ a year to individual members of supporting institutions.

Subscriptions, orders for back numbers, and changes of address should be sent to Pacific Journal of Mathematics, 103 Highland Boulevard, Berkeley, California, 94708.

PUBLISHED BY PACIFIC JOURNAL OF MATHEMATICS, A NON-PROFIT CORPORATION Printed at Jerusalem Academic Press, POB 2390, Jerusalem, Israel.

\section{Copyright (C) 1976 Pacific Journal of Mathematics} All Rights Reserved 


\section{Pacific Journal of Mathematics}

\section{Vol. 66, No. 2 December, 1976}

Gerald A. Beer, Tax structures whose progressivity is inflation neutral..... 305

William M. Cornette, A generalization of the unit interval............. 313

David E. Evans, Unbounded completely positive linear maps on

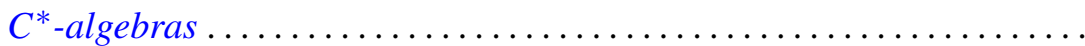

Hector O. Fattorini, Some remarks on convolution equations for

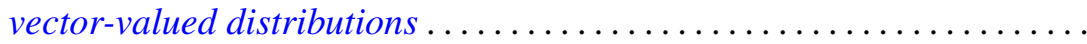

Amassa Courtney Fauntleroy, Automorphism groups of unipotent groups of

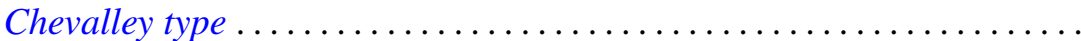

Christian C. Fenske and Heinz-Otto Peitgen, On fixed points of zero index in

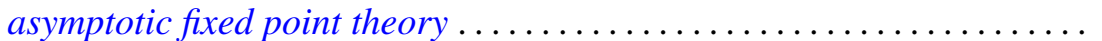

Atsushi Inoue, On a class of unbounded operator algebras. II ............

Herbert Meyer Kamowitz, The spectra of endomorphisms of algebras of

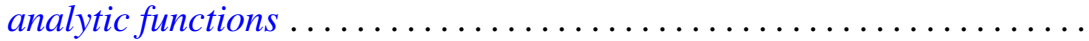

Jimmie Don Lawson, Embeddings of compact convex sets and locally compact cones ....................................

William Lindgren and Peter Joseph Nyikos, Spaces with bases satisfying certain order and intersection properties .....................

Emily Mann Peck, Lattice projections on continuous function spaces ...... 477

Morris Marden and Peter A. McCoy, Level sets of polynomials in $n$ real variables...

Francis Joseph Narcowich, An imbedding theorem for indeterminate Hermitian moment sequences......................

John Dacey O'Neill, Rings whose additive subgroups are subrings ...

Chull Park and David Lee Skoug, Wiener integrals over the sets bounded by sectionally continuous barriers .....................

Vladimir Scheffer, Partial regularity of solutions to the Navier-Stokes equations.

Eugene Spiegel and Allan Trojan, On semi-simple group algebras. II

Katsuo Takano, On Cameron and Storvick's operator valued function space integral 